\title{
Saint-Paul-Trois-Châteaux - Les Sablières
}

Opération préventive de diagnostic (2013)

Jean-Marc Lurol

\section{(2) OpenEdition}

\section{Journals}

Édition électronique

URL : https://journals.openedition.org/adlfi/14836

ISSN : 2114-0502

Éditeur

Ministère de la Culture

Référence électronique

Jean-Marc Lurol, « Saint-Paul-Trois-Châteaux - Les Sablières » [notice archéologique], ADLFI.

Archéologie de la France - Informations [En ligne], Auvergne-Rhône-Alpes, mis en ligne le 20 mai 2015,

consulté le 23 juillet 2021. URL : http://journals.openedition.org/adlfi/14836

Ce document a été généré automatiquement le 23 juillet 2021.

(c) ministère de la Culture et de la Communication, CNRS 


\section{Saint-Paul-Trois-Châteaux - Les Sablières}

Opération préventive de diagnostic (2013)

Jean-Marc Lurol

\section{NOTE DE L'ÉDITEUR}

Organisme porteur de l'opération : Inrap

1 Le diagnostic archéologique qui s'est déroulé, du 5 au 7 mars 2013 au quartier des Sablières (parcelles BY 116p et 117) a été occasionné par le projet d'agrandissement du lycée agricole à l'intérieur du clos des frères maristes. Dans ce quartier de la ville, classé en zone archéologique sensible, plusieurs interventions ont déjà eu lieu. Elles ont révélé à chaque fois la présence de vestiges, généralement datés de la période galloromaine, souvent bien conservés, mais profondément enfouis sous d'épaisses couches de colluvions sableuses provenant de la proche colline de Sainte-Juste qui domine la ville côté sud.

2 Sur ce terrain, l'ouverture de 17 sondages (7,2\% de l'emprise de l'étude), profonds en moyenne d'1, $5 \mathrm{~m}$ avec des tests réguliers jusqu'à $-2 \mathrm{~m}$, a révélé l'existence d'un niveau d'occupation dans l'angle sud-est de la parcelle BY $116 \mathrm{p}$ où il apparaît à $0,30 \mathrm{~m}$ de profondeur (alt. NGF : 98,10 m - sondage 3) et à 0,60 $\mathrm{m}$ de profondeur (alt. NGF : 97,50 m - sondage 2). Ce niveau se caractérise par une couche de sédiment sableux, de 0,10 à $0,15 \mathrm{~m}$ d'épaisseur, marquée par une pédogenèse brune. À l'intérieur de cette couche sont installés 6 fosses et un tronçon de mur. Le mobilier extrait à la fois du niveau de sol et des ensembles clos (fosses) a été daté des $\mathrm{V}^{\mathrm{e}}-\mathrm{VI}^{\mathrm{e}} \mathrm{s}$. apr. J.-C. Ce niveau d'occupation est spatialement restreint. Il n'est pas présent dans les autres espaces des parcelles étudiées ; toutefois, il peut très bien s'étendre vers l'est, hors emprise du projet, dans les parcelles voisines.

Dans les autres sondages, les vestiges observés se résument à une petite structure et une fosse. La première correspond sans doute à un drain. Son remplissage contenait des 
tessons de céramique datés du Haut-Empire. La seconde fosse très charbonneuse, ne renfermait pas de mobilier.

Dans l'ensemble des sondages, en dehors des indices d'occupation précédemment évoqués, la stratigraphie sur les deux premiers mètres d'épaisseur est constituée de dépôts très majoritairement sableux qui renferment en propositions variables des graviers et des cailloux de calcaire. Ces couches sableuses correspondent à des dépôts de pente (colluvions) qui proviennent de la colline de Sainte-Juste. Ces recouvrements ont déjà été observés lors des différentes interventions archéologiques effectuées dans ce secteur de la ville. Leur mise en place sur ce site reste difficile à dater, car toutes les couches de sable observées dans les sondages sont archéologiquement stériles.

La présence du niveau d'occupation daté des $\mathrm{V}^{\mathrm{e}}-\mathrm{VI}{ }^{\mathrm{e}} \mathrm{s}$. apr. J.-C., dans l'angle sud-est de la parcelle BY 116p, apporte cependant des informations intéressantes sur la mise en place des couches de sable. On peut admettre ainsi que, dans ce secteur du site, les horizons sableux sont antérieurs au début du haut Moyen Âge. Par contre, on ne peut pas appliquer cette interprétation pour les autres espaces du site où ce niveau n'est pas retrouvé. En effet, il est difficile d'établir avec certitude des équivalences entre les couches de sable observées dans les différents sondages, même si elles présentent des similitudes sédimentaires.

6 Un niveau d'occupation gallo-romain du Haut-Empire peut-il se trouver sous les sables à plus de $2 \mathrm{~m}$ de profondeur, comme cela est attesté sur les sites archéologiques voisins des Fouges (Landry 2010), des Sablières villa Augusta, du Pialon (maison de retraite et groupe scolaire : Ronco 2002) et des Fleuriades (Réthoré 2008) ? Cela ne semble guère possible dans le secteur où se trouve le niveau du début du haut Moyen Âge, car il faudrait envisager alors la mise en place de 1,5 $\mathrm{m}$ de dépôts sableux en moins de trois siècles et expliquer les raisons d'une telle érosion de la colline Sainte-Juste.

7 Dans les autres parties de l'emprise étudiée, la question reste posée. Pour ce secteur, le seul vestige daté (céramique du Haut-Empire) est le drain retrouvé à $1 \mathrm{~m}$ de profondeur dans le sondage 8. Mais il s'agit uniquement d'un élément isolé, spatialement et stratigraphiquement, qui ne peut à lui seul marquer un niveau d'occupation. Autre remarque : s'il existe un niveau d'occupation sous les sables, il est alors surprenant que ces couches de sable ne contiennent aucun artefact en position secondaire.

Reste possible l'hypothèse d'un aménagement en terrasses sur cet espace au cours du Haut-Empire (idée évoquée pour le site des Fouges) avec un étagement des niveaux de sols de circulation, qui pourrait en partie expliquer des enfouissements profonds des vestiges. Mais, dans ce cas, on devrait aussi les repérer par endroits à des profondeurs moins importantes. 


\section{INDEX}

lieux https://ark.frantiq.fr/ark:/26678/pcrtSEeAipsBlD, https://ark.frantiq.fr/ark:/26678/ crtB8WDyqd6u9, https://ark.frantiq.fr/ark:/26678/pcrtKgdLgiLWKX, https://ark.frantiq.fr/ark:/ 26678/pcrtP0VJP5NiOZ

nature https://ark.frantiq.fr/ark:/26678/pcrtWWQS75V5Bc

Année de l'opération : 2013

chronologie https://ark.frantiq.fr/ark:/26678/pcrtxT02uJOogm, https://ark.frantiq.fr/ark:/ 26678/pcrtof7EHNsS2e, https://ark.frantiq.fr/ark:/26678/pcrtwopiLhK1Ek, https://

ark.frantiq.fr/ark:/26678/pcrtZTmusVUU24, https://ark.frantiq.fr/ark:/26678/

pcrtIkSWVMVuqB, https://ark.frantiq.fr/ark:/26678/pcrtH5r3FYBpwe

sujets https://ark.frantiq.fr/ark:/26678/pcrtms2OAv82PY, https://ark.frantiq.fr/ark:/26678/ pcrtKJVpuP3AET, https://ark.frantiq.fr/ark:/26678/pcrtM9HMWQTGJV, https://ark.frantiq.fr/ ark:/26678/pcrtDpSNLz1mSr, https://ark.frantiq.fr/ark:/26678/pcrtIxHmbVwDYW, https:// ark.frantiq.fr/ark:/26678/pcrtIMsuGORWTs, https://ark.frantiq.fr/ark:/26678/pcrt1DMOWvDF4j, https://ark.frantiq.fr/ark:/26678/pcrthQAINOXOGB, https://ark.frantiq.fr/ark:/26678/ pcrtrrOj4xoA0D, https://ark.frantiq.fr/ark:/26678/pcrt2WF3gcWDUM, https://ark.frantiq.fr/ ark:/26678/pcrtthlTfnSHQp

\section{AUTEURS}

JEAN-MARC LUROL

Inrap 